\title{
Multimedia Mining RESEARCH - AN OVERVIEW
}

\author{
Dr. S.Vijayarani ${ }^{1}$ and Ms. A.Sakila ${ }^{2}$ \\ ${ }^{1}$ Assistant Professor, Department of Computer Science, Bharathiar University, \\ Coimbatore. \\ ${ }^{2}$ M.Phil Research Scholar, Department of Computer Science, Bharathiar University, \\ Coimbatore.
}

\begin{abstract}
Multimedia data mining is a popular research domain which helps to extract interesting knowledge from multimedia data sets such as audio, video, images, graphics, speech, text and combination of several types of data sets. Normally, multimedia data are categorized into unstructured and semi-structured data. These data are stored in multimedia databases and multimedia mining is used to find useful information from large multimedia database system by using various multimedia techniques and powerful tools. This paper provides the basic concepts of multimedia mining and its essential characteristics. Multimedia mining architectures for structured and unstructured data, research issues in multimedia mining, data mining models used for multimedia mining and applications are also discussed in this paper. It helps the researchers to get the knowledge about how to do their research in the field of multimedia mining.
\end{abstract}

Keywords: Data Mining, Multimedia Mining, Architectures, Applications, Models

\section{INTRODUCTION}

Multimedia data mining is used for extracting interesting information for multimedia data sets, such as audio, video, images, graphics, speech, text and combination of several types of data set which are all converted from different formats into digital media [18]. Multimedia mining is a subfield of data mining which is used to find interesting information of implicit knowledge from multimedia databases. Multimedia data are classified into five types; they are (i) text data, (ii) Image data (iii) audio data (iv) video data and (v) electronic and digital ink [2]. Text data can be used in web browsers, messages like MMS and SMS. Image data can be used in art work and pictures with text still images taken by a digital camera. Audio data contains sound, MP3 songs, speech and music. Video data include time aligned sequence of frames, MPEG videos from desktops, cell phones, video cameras [17]. Electronic and digital ink its sequence of time aligned $2 \mathrm{D}$ or $3 \mathrm{D}$ coordinates of stylus, a light pen, data glove sensors, graphical, similar devices are stored in a multimedia database and use to develop a multimedia system.

Since 1960s the research in the field of multimedia has initiated for combining different multimedia data into one application when text and images were combined in a document. During the research and development process of video synchronization of audio and animation was completed using a timeline to specify when they should be played [2]. The difficulties of multimedia data capture, storage, transmission and presentation have been explored in the middle of 1990s where the multimedia standards MPEG-4, X3D, MPEG-7 and MX have continued to grow. These are reformed and clearly handled sound, images, videos, and 3-D (three-dimension) objects that combined by events, synchronization, scripting languages which describe the content of any multimedia object [5]. For multimedia distribution and database applications different

DOI : $10.5121 /$ ijcga.2015.5105 
algorithms are required. Such a database can be queried, for example, with the SQL multimedia and application packages known as SQL/MM.

Multimedia database system includes a multimedia database management system (MMDBMS) which handles and provides foundation for storing, manipulating and retrieving multimedia data from multimedia database [4]. Multimedia data consists of structured data and unstructured data such as audio, video, graphs, images and text media.

The remaining portion of the paper is organized as follows. Section 2 presents the categories of multimedia data mining. Section 3 gives applications of multimedia mining. Multimedia data mining process is discussed in Section 4. Section 5 provides the multimedia architectures. Multimedia mining models are given in Section 6. Research issues are discussed in Section 7. Section 8 gives the conclusion.

\section{CATEGORIES OF MULTIMEDIA DATA MINING}

The multimedia data mining is classified into two broad categories as static media and dynamic media. Static media contains text (digital library, creating SMS and MMS) and images (photos and medical images). Dynamic media contains Audio (music and MP3 sounds) and Video (movies). Multimedia mining refers to analysis of large amount of multimedia information in order to extract patterns based on their statistical relationships. Figure 1 shows the categories of multimedia data mining.

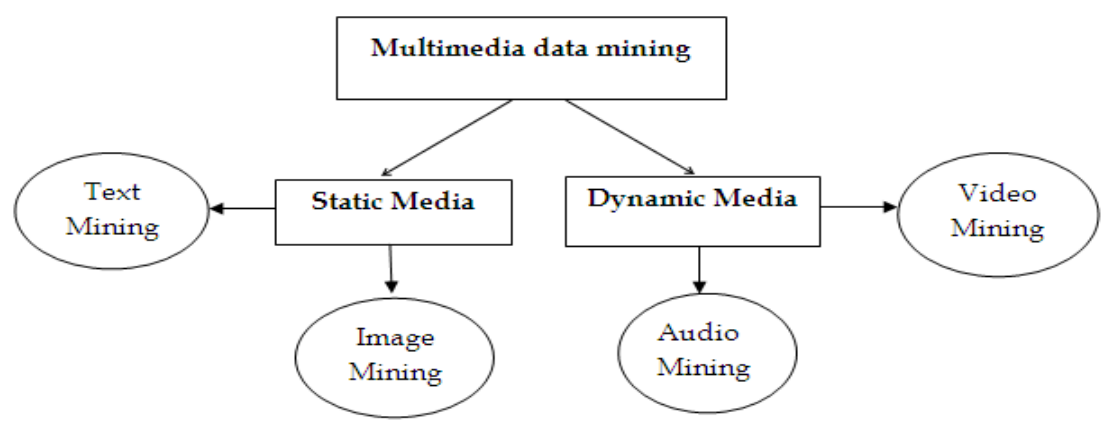

Figure 1. Categories of Multimedia Data Mining

\section{Text mining}

Text Mining also referred as text data mining and it is used to find meaningful information from the unstructured texts that are from various sources. Text is the foremost general medium for the proper exchange of information [3]. Text Mining is to evaluate huge amount of usual language text and it detects exact patterns to find useful information.

\section{Image mining}

Image mining systems can discover meaningful information or image patterns from a huge collection of images. Image mining determines how low level pixel representation consists of a raw image or image sequence can be handled to recognize high-level spatial objects and relationship [14]. It includes digital image processing, image understanding, database, AI and so on. 


\section{Video Mining}

Video mining is unsubstantiated to find the interesting patterns from large amount of video data; multimedia data is video data such as text, image, and metadata, visual and audio. The processing are indexing, automatic segmentation, content-based retrieval, classification and detecting triggers. It is commonly used in various applications like security and surveillance, entertainment, medicine, sports and education programs [15].

\section{Audio mining}

Audio mining plays an important role in multimedia applications, is a technique by which the content of an audio signal can be automatically searched, analyzed and rotten with wavelet transformation. Band energy, frequency centroid, zero crossing rate, pitch period and band-width are often used features for audio processing [2]. It is generally used in the field of automatic speech recognition, where the analysis efforts to find any speech within the audio [11].

\section{APPLICATIONS OF MULTIMEDIA MINING}

There are different kinds of applications [1] [8] of multimedia data mining, some of which are as follows:

Digital Library: The collection of digital data are stored and maintained in digital library, which is essential to convert different formats of digital data into text, images, video, audio, etc.

Traffic Video Sequences: In order to determine important but previously unidentified knowledge from the traffic video sequences, the detailed analysis and mining to be performed based on vehicle identification, traffic flow, and queue temporal relations of the vehicle at intersection. This provides an economic approach for regular traffic monitoring processes.

Medical Analysis: Multimedia mining is primarily used in the medical field and particularly for analyzing medical images. Various data mining techniques are used for image classification. For example, Automatic 3D delineation of highly aggressive brain tumors, Automatic localization and identification of vertebrae in 3D CT scans, MRI Scans, ECG and X-Ray.

Customer Perception: It contains details about customers opinions, products or services, customers complaints, customers preferences, and the level of customer's satisfaction of products or services which are collected together. Many companies have call centers that receives telephone calls from the customers. The audio data serves as topic detection, resource assignment and evaluation of quality of services.

Media Making and Broadcasting: Radio stations and TV channels creates broadcasting companies and multimedia mining can be applied to monitor their content to search for more efficient approaches and improve their quality.

Surveillance system: It consists of collecting, analyzing, summarizing audio, video or audio visual information about specific areas like government organizations, multi-national companies, shopping malls, banks, forest, agricultural areas and highways etc. The main use of this technology in the field of security hence it can be utilized by military, police and private companies since they provide security services. 


\section{MULTIMEDIA DATA MINING PROCESS}

Figure 2 shows present architecture which includes the types of multimedia mining process [19]. Data Collection is the initial stage of the learning system; Pre-processing is to extract significant features from raw data, it includes data cleaning, transformation, normalization, feature extraction, etc. Learning can be direct, if informative types can be recognized at pre-processing stage. Complete process depends extremely on the nature of raw data and difficulty's field. The product of pre-processing is the training set. Specified training set, a learning model has to be selected to learn from it and make multimedia model is more constant.

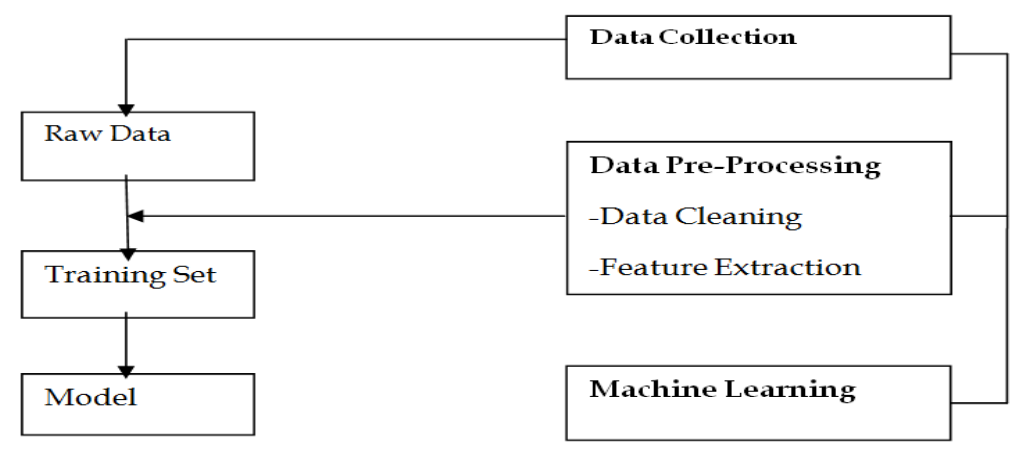

Figure 2. Multimedia Mining Process

\section{Converting Un-structured data to structured data:}

Data resides in fixed field within a record or file is called structured data and these data are stored in sequential form. Structured data has been easily entered, stored, queried and analyzed. Unstructured data is bit stream, for example pixel representation for an image, audio, video and character representation for text [1]. These sorts of files may have an internal structure, they are still considered "unstructured" because the data they contain does not fit neatly in a database. For example, image and video of different objects has some similarity - each represents an interpretation of a building - but then without clear structure.

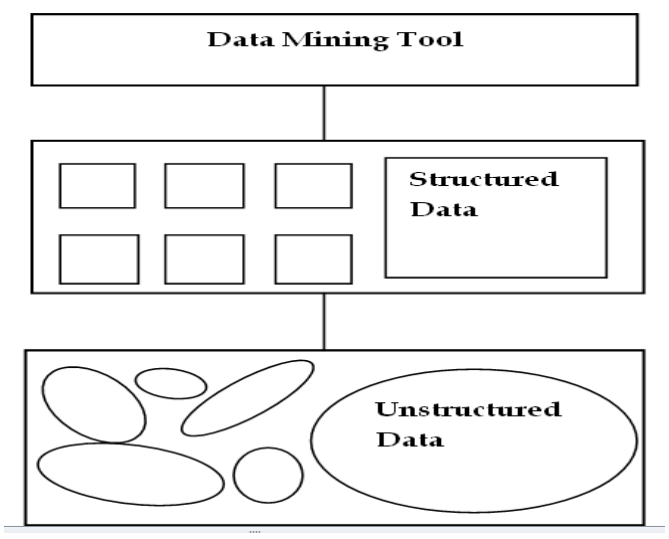

Figure 3. Unstructured Data to Structured Data Conversion 
Current data mining tool operate on structured data, which resides in huge volume of relational database while data in multimedia databases are semi-structured or un-structured. Hence, the semi-structured or unstructured multimedia data is converted into structured one, and then the current data mining tools are used to extract the knowledge. A difference between unstructured data and structured data mining is the sequence or time element. The architecture of converting unstructured data to structured data and it is used for extracting information from unstructured database is shown in Figure 3. Then data mining tools are applied to the stored structured databases.

\section{ARCHITECTURES FOR MULTIMEDIA DATA MINING}

Multimedia mining architecture is given in Figure 4. The architecture has several components. Important components are (1) Input (2) Multimedia Content (3) Spatiotemporal Segmentation (4) Feature Extraction (5) Finding the similar Patterns and (6) Evaluation of Results.

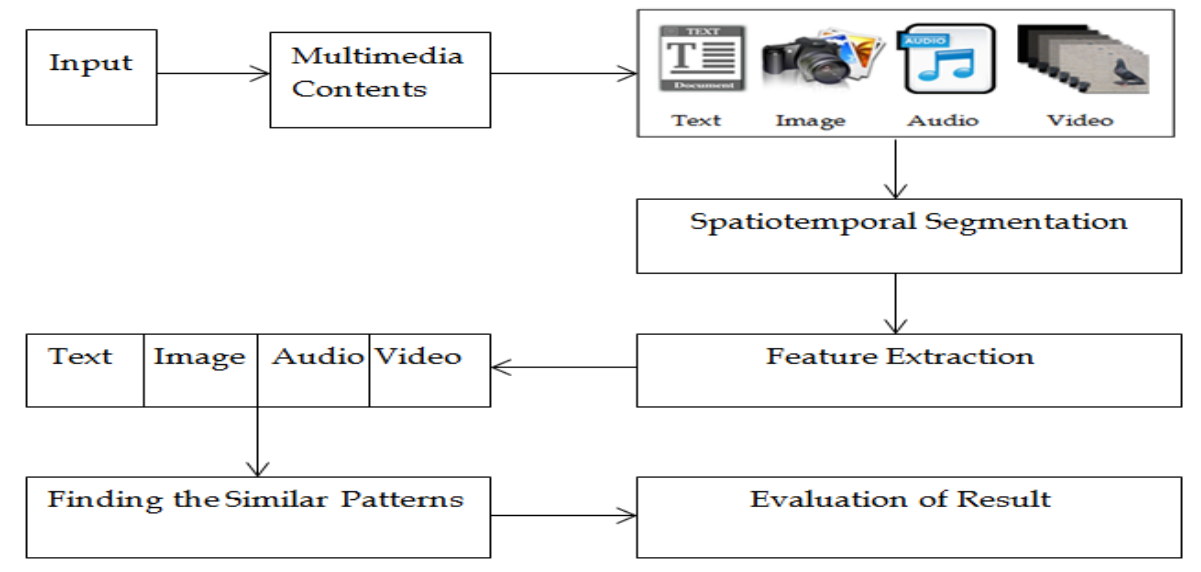

Figure 4. Multimedia Data mining Architecture

1. Input stage comprises which multimedia database is used for finding the patterns and to perform data mining process.

2. Multimedia Content is the data selection stage which requires the user to select the databases, subset of fields or data to be used for data mining.

3. Spatio-temporal segmentation is nothing but moving objects in image sequences in the videos and it is useful for object segmentation.

4. Feature extraction is the pre-processing step that involves integrating data from various sources and making choices regarding characterizing or coding certain data fields to serve when inputs to the pattern finding stage. Such representation of choices is required because certain fields could include data at various levels and not considered for finding the similar pattern stage. In MDM the preprocessing stage is significant since the unstructured nature of multimedia records.

5. Finding the similar pattern stage is the heart of the whole data mining process. The hidden patterns and trends in the data are basically uncovered in this stage. Some approaches of finding similar pattern stage contain association, classification, clustering, regression, time-series analysis and visualization. 
6. Evaluation of Results is a data mining process used to evaluate the results and this is important to determine whether prior stage must be revisited or not. This stage consists of reporting and makes use of the extracted knowledge to produce new actions or products and services or marketing strategies [8].

\section{MODELS FOR MULTIMEDIA MINING}

The models which are used to perform multimedia data are very important in mining. Commonly four different multimedia mining models have been used. These are classification, association rule, clustering and statistical modeling.

\subsection{Classification}

Classification is a technique for multimedia data analysis, can learn from every property of a specified set of multimedia. It is divided into a predefined class label, so as to achieve the purpose of classification. Classification is the process of constructing data into categories for its better effective and efficient use, it creates a function that well-planned data item into one of many predefined classes, by inputting a training data set and building a model of the class attribute based on the rest of the attributes. Decision tree classification has a perceptive nature that the users conceptual model without loss of exactness. Hidden Markov Model used for classifying the multimedia data such as images and video as indoor-outdoor games [6].

\subsection{Association Rule}

Association Rule is one of the most important data mining technique which helps to find relations between data items in huge databases. There are two different types of associations in multimedia mining: association between image content and non-image content features [1]. Mining the frequently occurring patterns between different images becomes mining the repeated patterns in a set of transactions. Multi-relational association rule mining is used to display the multiple reports for the same image. In image classification also multiple level association rule techniques are used.

\subsection{Clustering}

Cluster analysis divides the data objects into multiple groups or clusters. Cluster analysis combines all objects based on their groups. Clustering algorithms can be divided into several methods they are hierarchical methods, density-based methods, grid-based methods, and modelbased methods, k-means algorithm and graph based model [3]. In multimedia mining, clustering technique can be applied to group similar images, objects, sounds, videos and texts.

\subsection{Statistical Modeling}

Statistical mining models are used to regulate the statistical validity of test parameters and have been used to test hypothesis, undertake correlation studies and transform and make data for further analysis. This is used to establish links between words and partitioned image regions to form a simple co-occurrence model [9].

\section{RESEARCH ISSUES IN MULTIMEDIA MINING}

Major Issues in multimedia data mining contains content based retrieval, similarity search, dimensional analysis, classification, prediction analysis and mining associations in multimedia data [7]. 


\subsection{Content based retrieval and Similarity search}

Content based retrieval in multimedia is a stimulating problem since multimedia data is required for detailed analysis from pixel values [6]. We considered two main families of multimedia retrieval systems i.e. similarity search in multimedia data.

(1) Description-based retrieval system created indices and make object retrieval, based on image descriptions, for example keywords, captions, size, and time of creation.

(2) Content-based retrieval system supports retrieval on the image content, for example color histogram, texture, shape, objects and wavelet transforms.

Use of content-based retrieval system: Visual features to index images and promotes object retrieval based on feature similarity; it is very desirable in various applications [9]. These applications which include diagnosis, weather prediction, TV production and internet search engines for pictures and e-commerce.

\subsection{Multidimensional Analysis}

In order to perform multidimensional analysis of large multimedia databases, multimedia data cubes may be designed and constructed in a method similar to that for traditional data cubes from relational data. A multimedia data cube can have additional-dimensions and measures for multimedia data, such as color, texture, and shape. A multimedia data cube has several dimensions. Examples are: size of the image or video in bytes; width and height of the frames, creating two dimensions, date on which image or video was created or last modified, format type of the image or video, frame sequence duration in seconds, Internet domain of pages referencing the image or video, the keywords like a color dimension and edge orientation dimension.

Multimedia data mining system prototype is called MultiMediaMiner which is the extension of DBMiner system handles multimedia data. The Image Excavator component of MultiMediaMiner uses image contextual information, like HTML tags in Web pages, to derive keywords [10]. By navigating on-line directory structures, like Yahoo! directory, it is possible to build hierarchies of keywords mapped on the directories in which the image was found.

\subsection{Classification and Prediction Analysis}

Classification and predictive analysis has been used for mining multimedia data particularly in scientific analysis like astronomy, seismology, and geo-scientific analysis. Decision tree classification is an important data mining method in reported image data mining applications. For example, consider the sky images which has been carefully classified by astronomers as the training set, it can create models for the recognition of galaxies, stars and further stellar objects, based on properties like magnitudes, areas, intensity, image moments and orientation. The image data are frequently in large volumes and needs substantial processing power, for example, parallel and distributed processing. Image data mining classification and clustering are carefully connected to image analysis and scientific data mining and hence many image analysis techniques and scientific data analysis methods could be applied to image data mining [10]. 


\subsection{Mining Associations in Multimedia Data}

Association rules involving multimedia objects have been mined in image and video databases. Three categories can be observed:

1. Associations between image content and non-image content features

2. Associations among image contents that are not related to spatial relationships

3. Associations among image contents related to spatial relationships

The associations between multimedia objects, we can treat every image as a transaction and find commonly occurring patterns among different images. First, an image contains multiple objects, each with various features such as color, shape, texture, keyword and spatial locations, so that there can be a huge number of possible associations. Second, a picture containing multiple repeated objects is an essential feature in image analysis, recurrence of the similar objects should not be ignored in association analysis. Third, to find the associations between the spatial relationships and multimedia images and this can be used for discovering object associations and correlations [9].

\section{CONCLUSION}

Multimedia mining is one of the important and challenging research domains in the field of computer science. Most of the researchers are interested to do their research work in the field of multimedia mining. Many challenging research problems are available in multimedia mining. These problems can be solved by developing new algorithms, concepts and techniques for extracting hidden knowledge from the multimedia data bases. This paper discussed the multimedia mining basic concepts, essential characteristics, architectures, models and applications. Emerging and open research issues in multimedia mining also described in this paper.

\section{REFERENCES}

[1] Manjunath T.N, Ravindra S Hegadi, Ravikumar GK, "A Survey on Multimedia Data Mining and Its Relevance Today" IJCSNS International Journal of Computer Science and Network Security, VOL.10 No.11, November 2010.

[2] Sarla More, Durgesh Kumar Mishra, "Multimedia Data Mining: A Survey" Pratibha: International Journal of science, spirituality, business and technology (ijssbt), vol. 1, no.1, march 2012 issn (print) 2277-7261.

[3] Manjunath R, S. Balaji, "Review and Analysis of Multimedia Data Mining Tasks and Models"InternationalJournal of Innovative Research in Computer and Communication Engineering Vol.2, Special Issue 2, May 2014.

[4] Bhavanithuraisingham, "Managing and mining multimedia Databases" International Journal on Artificial Intelligence Tools Vol. 13, No.3 (2004) 739-759.

[5] JanuszSwierzowicz,"Multimedia Data Mining Trends and Challenges".

[6] Yu H, Wolf, Scenic classification methods for image and video databases. In In SPIE International Conference on Digital Image Storage and Archiving Systems, Vol. 2606,1995, pp. 363-371.

[7] Mittal, Ankush, "An overview of multimedia content-based retrieval strategies", Publication: Informatica, October 12006.

[8] Valery A. Petrushin and Latifur Khan, "Multimedia Data Mining and Knowledge Discovery", Springer, 2007 pp. 3- 17

[9] Jiawei Han, MichelineKamber "Data Mining: Concepts and Techniques" Published by Morgan Kaufmann, 2001. 
[10] Mori Y, Takahashi H, Oka R. Image-to-word transformation based on dividing and vector quantizing images with words. In: MISRM'99 First International Workshop on Multimedia Intelligent Storage and Retrieval management, 1999

[11] http://en.wikipedia.org/wiki?curid=14004969

[12] Chapter 25 Mining Multimedia Databases, Data Mining Techniques, Arun k pujari 1st Edition, Data warehousing, Data Mining and OLAP, Alex Berson ,smith.j. Stephen,

[13] Data Mining Concepts and Techniques, Jiawei Han and MichelineKamber, Data Mining Introductory and Advanced topics, Margaret H Dunham PEA, The Data Warehouse lifecycle toolkit, Ralph Kimball Wiley student Edition

[14] Ordenoz C, Omiecinski E. "Discovering association rules based on image content" In:ADL '99: Proceedings of the the IEEE Forum on Research and Technology Advances in Digital libraries.Washington, DC: IEEE Computer Society; 1999, p.38.

[15] V. Vijayakumar, R. Nedunchezhian "A study on video data mining", International Journal of Multimedia Information Retrieval, October 2012, Volume 1, Issue 3, pp 153-172, Publisher Springer-Verlag.

[16] http://www.comp.nus.edu.sg/ chidansh

[17] Janusz Swierzowicz "Multimedia Data Mining Concept".

[18] Madiha Waris Farooque Azam Abdul Wahab Muzaffar A Survey of Issues in Multimedia Databases international Journal of Computer Applications (0975 - 8887) Volume 46 - No.7, May 2012

[19] S. Kotsiantis, D. Kanellopoulos, P. Pintelas, Multimedia Mining, (2004). 'Multimedia mining', WSEAS Transactions on Systems,. Vol. 3, No. 10, pp.3263-3268.

$\mathrm{Yu} \mathrm{H}$, Wolf, Scenic classification methods for image and video databases. In In SPIE International Conference on Digital Image Storage and Archiving Systems, Vol. 2606,1995,pp. 363-371

\section{BIOGRAPHY}

Dr. S. Vijayarani has completed MCA, M.Phil and Ph.D in Computer Science. She is working as Assistant Professor in the School of Computer Science and Engineering, Bharathiar University, Coimbatore. Her fields of research interest are data mining, privacy and security issues in data mining and data streams. She has published papers in the international journals and presented research papers in international and national conferences.

Ms. A. Sakila has completed M.Sc in Computer Science. She is currently pursuing her M.Phil in Computer Science in the School of Computer Science and Engineering, Bharathiar University, Coimbatore. Her fields of interest are Data Mining and Multimedia Mining.
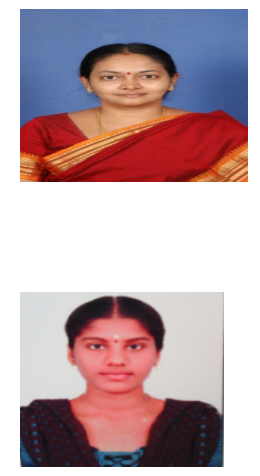